\title{
FUNCTIONS OF PLANT'S BZIP TRANSCRIPTION FACTORS
}

\author{
Zulfiqar Ali, ${ }^{1,3}$, , Syeda Samara Sarwat ${ }^{1}$, Ihsan Karim¹, Rabia Faridi', Muhammad Jafar Jaskani² \\ and Asif Ali Khan ${ }^{1}$
}

\author{
${ }^{1}$ Department of Plant Breeding and Genetics, University of Agriculture, Faisalabad-38040, Pakistan; ${ }^{2}$ Institute of \\ Horticultural Sciences, University of Agriculture, Faisalabad-38040, Pakistan; ${ }^{3}$ Department of Plant Breeding and \\ Genetics, Muhammad Nawaz Shareef Univeristy of Agriculture, Multan-60000. Pakistan. \\ "Corresponding author's e-mail: zulfiqar_ali@uaf.edu.pk
}

\begin{abstract}
Basic leucine zipper protein refers to a grand transcriptional factor family of all eukaryotes that is involved in various developmental and stress responses like flower development, plant height, maturation of seeds, biotic and abiotic stress signaling. About 127 bZIPs in Arabidopsis, 70 in cotton (Gossypium hirsutum), 266 in soybean (Glycine max), 47 in tobacco (Nicotiana tabacum), 70 in tomato (Solanum lycopersicum), 140 in rice, 102 in wheat and 218 in maize have been discovered. In this review, the classification of bZIP is according to the binding specificities of bZIPs as well as their role in transcription. Keywords: Basic leucine zipper, abscisic acid, cold signalling, pathogen response, core sequence
\end{abstract}

\section{INTRODUCTION}

The basic leucine zipper termed as bZIP protein is a grand transcriptional regulator's family (Umezawa et al., 2006) especially in eukaryotes. The proteins of bZIP normally consist of a bZIP domain having two fundamental characteristics: basic region and dimerization region. Basic region binds to DNA and dimerization region form homo as well as heterodimers. In the basic region, there is a nuclear localization signal which follows on an invariant $\mathrm{N}-\mathrm{x} 7-\mathrm{R} / \mathrm{K}$ motif which binds to DNA. Moreover, there is a repeat of a heptad of leucine located accurately nine amino acids along the C-terminus, giving rise to an amphipathic helix. Plant bZIP transcription factors favorably bind to ACGT core DNA sequences like G-box (CACGTG), C-box (GACGTC), A-box (TACGTA) (Jakoby et al., 2002), PB-like (TGAAAA) ABRE (ABA responsive element i.e. CCACGTGG) (Liao et al.,
2008) and GLM (GCN4-like motif i.e. GTGAGTCAT) (Holdsworth et al., 1995) motif occur in promoters of many stress signaling genes. However, some non-palindromic sites are also found in bZIP (Zhang et al., 2008).

Mechanism of action of bZIP transcription factors: The transcription factors of bZIP family bind to promoter sequence of selected genes as they are characterized to homodimerize as well as heterodimerize. A transcription factor is termed as regulon when it is involved in the regulation of a great range of genes' expression by binding to the cis-acting regions of the promoters (Nakashima et al., 2009). All bZIP family members are evolutionarily interrelated and conserved. Hence, they share a fundamental integrated construction encompassing a basic zipper (bZIP) domain (Fig. 1)

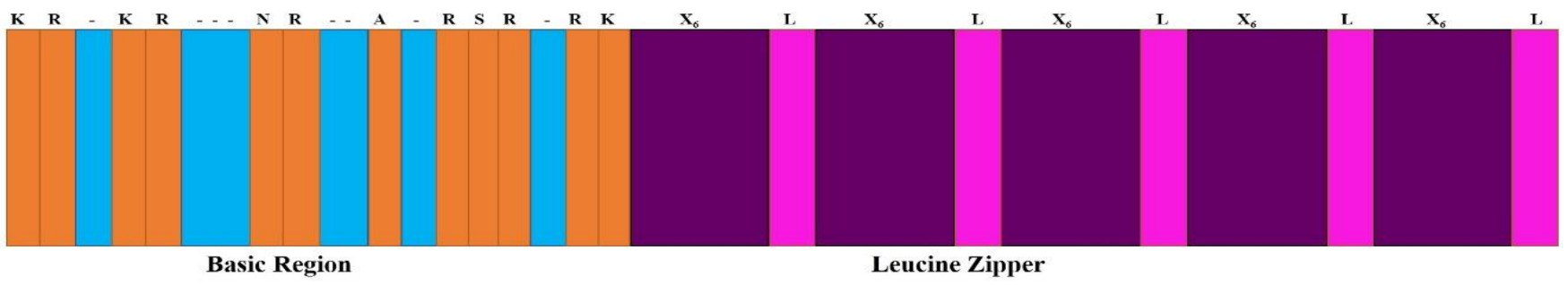

Figure 1. Basic leucine zipper motif showing basic region and leucine zipper region.

Leucine zipper is an extremely conserved protein as it contains a motif of five heptads of amino acids placed sequentially with a leucine at seventh spot. Leucine is highly stabilizing as compared to other amino acids (Vincentz et al., 2001). The leucine zippers switch the homo- and heteroconnotation of bZIP members. They have incessant $\alpha$-helices in the framework of a dimer which show characteristic curling around the opposite homo or heterodimer, formulating a dimeric and parallel twisted coil with a trivial twist towards left. The basic regions of bZIP domain remain at the $\mathrm{N}$ termini and hence it becomes able to attach at the promoter regions by making a parallel in the major groove of DNA (see 
Fig. 2, Jakoby et al., 2002). Recent researches through isothermal titration calorimetry (ITC) and analytical LASER scattering (ALS) technique have revealed that five heptads in leucine zippers are most important for the bZIP domain dimerization (Selvaraj et al., 2010). It has also been elucidated that the sequential C-terminal truncation of residues reaching up to each leucine vividly liaises the binding of bZIP domains to DNA (Seldeen et al., 2010).

Regulatory functions of bZIP factors: Transcription factors regulate a lot of functions in plants in response to stressful conditions (Allen, 2012) by producing different types of proteins (Singh et al., 2002) e.g. anti-oxidants, dehydrins and osmolytes synthesizing enzymes (Bartels and Sunkar, 2005). Like other transcription factor families, most of bZIP proteins play intersecting role due to which the study of mutant individual's phenotype might become obscure; making the recognition of all $b Z I P$ genes a compulsion for the identification of functions of bZIP proteins at individual level (Jakoby et al., 2002). They are most common in plants to regulate a variety of phenomena like germination, photomorphogenesis, formation of flowers, floral development, maturation of seed, and are also important for plants in signaling of stress and hormones. Plant bZIP proteins have been extensively explored and their functions seem to be more complex and expanded than other transcriptional factors (Wei et al., 2010). Abssisic acid (ABA) responsive element (ABRE) is one of the recognized gene expression regulatory systems dependent upon ABA production. All the genes of plants using ABRE system require bZIP for their expression (Liao et al., 2008).

Plant bZIP interactions with $\boldsymbol{A B A}$ : The $\mathrm{ABA}$ is an important plant hormone which shows a vital role in germination and maturation of seed, in addition to arbitrating adaptive responses to abiotic stresses (Wei et al., 2010) as ABA is produced in plants when exposed to any type of abiotic stress (Allen, 2012). Many genes that are activated by ABA signal may be initiated by exogenous application of ABA (Kang et al., 2002; Cheong et al., 2003). ABA improves plant's capability to survive in adverse environments and productivity (Liao et al., 2008). ABRE are cis-acting elements found in the upstream of ABA responding genes (ABFs) (Shen and Ho, 1995). Events of signal transduction during cold and drought stress are intersecting (Shinozaki et al., 2003) and C-repeat/dehydration-responsive element (CRT/DRE) interacts with ABRE-binding bZIP proteins to control the cold and drought responsive genes expression (Narusaka et al., 2003). ABA is also involved in enhancing the role of many genes like late-embryogenesis-abundant genes e.g., HVA1. The bZIP transcription factor TaABF1 is helpful in the signaling of ABA pathway which affects $H V A 1$ expression. Exploration of the interaction between $\mathrm{ABA}$ and
TaABF1 in the aleuronic cells of imbibing cereal grains showed that the TaABF1 and ABA are not additive in stimulation of the HVAl promoter (Keyser, 2010). However, abiotic stress response systems of genes may be dependent as well as independent of ABA signal like some genes are present that activate by water stress but they show no response to even exogenous application of ABA (Chinnusamy et al., 2004).

Binding specificities of bZIPs: The plant bZIPs are highly specific to their binding activity and their function totally depends upon their binding ability. Hence on the basis of specificity, all bZIPs can be divided into various groups.

Group1: This can also be termed as G-box binding factor (GBF) which includes the bZIPs that recognize ACGT sequence in DNA. A GBF group includes EMBP-1 proteins in maize, CPRF-1 in Parsley, TAF1 in tobacco and GBF1 of Arabidopsis and many more. More than 40 bZIP transcription factors bind to the G-box.

Group 2: This is not very versatile and grand as it includes RITA-1 in rice, CPRF-2 in parsley and opaque-2 proteins found in maize.

Group 3: TGA factors are referred as the third group of bZIP transcription factors that binds to the TGACGT core sequence of DNA. The third group includes tobacco TGA1 (Quatrano, 1996).

bZIP in different plants and their classification: Many bZIP proteins have been identified in various plants (Table 1) e.g. 127 bZIPs in Arabidopsis, 70 in cotton (Gossypium hirsutum), 266 in soybean (Glycine max), 47 in tobacco (Nicotiana tabacum), 70 in tomato (Solanum lycopersicum), 140 in rice, 102 in wheat and 218 in maize (Zea mays) have been discovered (For complete phylogeny please visit http://planttfdb.cbi.pku.edu.cn/). On the basis of sequences of amino acids found in basic and leucine zipper region, bZIPs are categorized into following groups (see Fig. 1, Fukazawa et al., 2000).

i. $\quad$ bZIPs in Arabidopsis: All bZIP transcription factors in Arabidopsis have been given a generic name AtbZIPl to AtbZIP75 (Table 1). This naming system is not according to a proper justification but gives a distinct identification to each $b Z I P$ gene. All identified bZIP genes and their structured nomenclature has been amalgamated into the MAtDB (MIPS Arabidopsis thaliana Database) where MIPS: Munich Information Center for Protein Sequences (Jakoby et al., 2002). The classes of Arabidopsis thaliana genes (AtbZIP) are as follow:

Group A: This consists of seven members: $A B I 5$, $A B F 2 / A R E B 1, A B F 4 / A R E B 2, A R E B 3, G B F 4, A B F 1$ and $A B F 3$. Abiotic stresses and $\mathrm{ABA}$ induces expression of abscisic acid binding factor $(A B F)$ also termed as ABA- 
responsive element-binding protein (AREB) (Liao et al., 2008) and ABA also initiates phosphorylation of AREB1/2. ABA induced phosphorylation is essential for AREB $1 / 2$ for induction of downstream sequences in casein kinase II phosphorylation. Hence it is concluded that ABA and stress are most likely to perform both transcriptional and posttranslational regulation as well as ABA signal transduction of group A-bZIP (Jakoby et al., 2002; Schutze et al., 2008). Plant bZIP transcription factors may lose their constancy due to phosphorylation. ABA- induced phosphorylation of AtABI5 enhances the steadiness of proteins and lessens its degradation. The same happens in HvABI5, AtABI5 homolog from barley. A unique ABI5-binding protein has been isolated, named ABI five binding protein (AFP) which is assumed to aim at ABI5 for its degradation mediated by ubiquitin. Still the relation of ABI5 phosphorylation and AFP has not been comprehended. One reason could be that ABI5 which is the phosphorylated form long hypocotyl 5 (Hy5) proteins is rather established in darkness. This is elucidated by the fragile interface of the ABI5 factor with the E3 ubiquitin ligase constitutive photo morphogenetic 1 (COP1), which degrades HY5 through proteasome. Proteasomes are the bodies degenerative to unnecessary proteins in the body. As we can see the unique role for HY5 and COP1 in Arabidopsis and identified unique in vivo characteristic HY5 genes, it has become interesting to examine whether the light regulated genes are highly specific in their function and the differential phosphorylation of HY5 might pave way for the classification of HY5 on the basis of different light qualities and responses to them (Schutze et al., 2008). ABF1 is expressed in vegetative tissues after cold treatment whereas ABF2, ABF3 and ABF4 are induced by salinity, drought and ABA (Table 2). Over expression of ABF3 and ABF4 leads to improved drought tolerance (Liao et al., 2008). A member of group-A of Arabidopsis assists in antagonistic stimulation of inflorescence, named as flowering locus D (FD). FD expresses in the tips of branches and interrelates with two genes terminal flower 1 (TFL1) and flowering locus T (FT) and it utilizes APETALA1 AP1 gene to promote flowering. FD is not an efficient flowering initiator, but when it associates with FT or TFL1, it is transmuted into a strong inflorescence activator and repressor, respectively (Schutze et al., 2008). AREB1 is an essential component of various abiotic stresses like drought, heat and salt as its over expression plays important role in glucose signaling (Liao et al., 2008).

Group B: This includes three bZIP members AtbZIP17, AtbZIP28 and AtbZIP49 which contain a putative transmembrane domain (TMD) and putative protease site-1 (S1P) which is a cleavage site in their luminal parts. AtbZIP17 has been reported to be involved in the unfolded protein response (UPR), and AtbZIP17 and AtbZIP28 are involved in various types of endoplasmic reticulum stress responses (Table 1,2)
(Schutze et al., 2008).

Group C: This group contains common plant regulatory factor 2 (CPRF2) and G-box binding factor1 also known as Histone binding factor $1(\mathrm{G} / \mathrm{HBF}-1)$ and has same structural features as Opaque $2(\mathrm{O} 2)$ transcription factors in many monocots. O2 interacts with prolamin-box binding factor (PBF) protein and controls seed storage protein production. CPFR2 and G/HBF-1 show responses to environmental stresses and pathogens. It is yet to be verified whether the contiguous Opaque2 homologues AtbZIP10 and AtbZIP25 also control the seed storage protein production in Arabidopsis (Jakoby et al., 2002).

AtbZIP10 is disseminated equally to the cytoplasm as well as the nucleus. Nuclear export activity of AtXPO1 (nuclear export receptor exportin 1) interacts with AtbZIP10 and a gene for lesion simulating disease 1 (LSD1). LSD1 is a zinc finger protein regarded as a negative controller of cell death induced by reactive oxygen species (ROS). N-terminal nuclear export sequence (NES) facilitates the collaborative activity of AtbZIP10 with AtXPO1, and its interaction with LSD1 is reconciled by a region in the $\mathrm{C}$ terminus. LSD1 covers the AtbZIP10 to eliminate its nuclear uptake. Being a repressor, LSD1 can inhibit the bZIP factors from binding to the DNA e.g. by attaching to AtbZIP10 in the nucleus (see Fig. 3, Schutze et al., 2008).

AtbZIP10 gets successful in formulating heterodimers with AtbZIP53, an S-group bZIP, when it has been accommodated in the nucleus. AtbZIP10 also starts interaction with non-bZIP proteins like $\mathrm{ABI} 3 . \mathrm{ABI} 3$ is a vital co-regulator of a developing seeds bZIP-dependent gene expression (Schutze et al., 2008). AtbZIP10 is unrestricted under oxidative stress conditions like pathogen attack, ROS conditions or salicylic acid (SA) treatment (Table 2). The exact mechanism of release of AtbZIP10 is yet to be known. AtbZIP10 is then moved to the nucleus where it can initiate the transcription of objective genes (see Fig. 2, Jakoby et al., 2002). AtbZIP10 functions as a basal defense and initiator of pathogen reaction. Hence it is repressor of oxidative cell death along with LSD1(Kaminaka et al., 2006).

Group D: Members of this gene group are involved to confer resistance against diseases and in the development of plant. These genes are also involved in transduction of different systemic signals e.g., SA and ethylene at the pathogenesis related (PR) promoter in response to pathogen infection (Jakoby et al., 2002). Members of the D group of Arabidopsis bZIP factors are also termed as TGAs, symbolizing the transcription factors binding to the core region of TGACGT in DNA. Orthologs of TGAs from other plant species play role in transcriptional activity during their plant's protection against diseases particularly in systemic acquired resistance (SAR). SAR is a broad spectrum, long-term safeguard against microbes and is initiated when plants are exposed to pathogens. When the pathogen has been identified, PR genes 
become transcriptionally stimulated by a mechanism of SA initiated at the sites of prime infection, and in related portions of plants. TGAs recognize the AS-1 (activation sequence-1)type cis-element in the promoter of PR genes. TGAs activate at the PR promoters upon the SA signaling and hence initiate the PR expression. The non-expressor-of-pathogenesisrelated-genes-1 protein (NPR1), synonym non-inducible immunity 1 (NIM1) suppresses the DNA binding and transcriptional capabilities of TGAs. When the SA signals are lacking, NPR1 gathers as a heavy molecular weight molecule in the cytoplasm, maintained by intermolecular cysteine bridges. SA treatment reconciles any modification in the redox condition of the plant cell. Hence it resolves cysteine bridges and performs the nuclear import of NPR1. On the other hand TGA interfaces with NPR1 in the nucleus, trailed by PR gene activation. Thorough analysis of SAR initiation has revealed that DNA-TGA2-NPR1 formulate a ternary complex, which acts as an enhanceosome. Function of DNATGA2-NPR1 complex is dependent on SA. Analysis of SA treated cell's nucleus, disclosed that NPR1 and TGA2 can initiate gene expression after treatment with SA in transitorily altered Arabidopsis leaves, whereas TGA2 alone could not perform so (Schutze et al., 2008). It was also clear that BTBPOZ (BTB: bric-a-brac tramtrack broad and POZ: Pox virus and zinc finger) interaction domain was compulsory for the co-activator function of NPR and the oxidation of Cys 521 and Cys 529. In actual, TGA2 is a repressor of PR gene expression in the existence of other TGAs. Hence it is postulated that TGA2 works as transcriptional activator as well as a repressor. The control of SAR through TGAdependent signaling mechanisms has been assumed alike in the whole plant kingdom after the discovery of NPR. In rice, about four TGA-like factors operate including rTGA2.1 and rTGA2.2. They act together with rice NPR1 ortholog NH1 and Arabidopsis NPR1. It has been observed that overexpression of both rice NH1 and Arabidopsis NPR1 augments the resistance of the transgenic plants to bacterial disease Xanthomonas oryzae PV. Oryzae (Xoo). At the same time there arises a negative function by rTGA2.1 in rice against bacterial pathogens. Removal of rTGA2.1 function reduces the disease symptoms in the infected rice plants with Xoo pathogen (see Fig 2, Schutze et al., 2008).

There are two more genes which perform developmental progressions; AtbZIP46/Perianthia (PAN) controls inflorescence in Arabidopsis and liguleless 2 controls the formation of blade sheath edges in maize. Arabidopsis NPR1gene is a homolog of BLADE-ON-PETIOLE1 (BOP1) and $\mathrm{BOP} 2$. BOP1 and BOP2 function is redundant to check disproportionate growth and prefiguring of leaves and flowers. In the developmental processes only BOP1 and BOP2 act together with a TGA factor (PAN); NPR1 is not involved. Detailed exploration of genetic mechanisms has revealed that interaction of BOP proteins with PAN monitors the asymmetric leaf growth and as the NPR1- related signaling is similar to that for controlling the SAR is also used for the organ formulation (Schutze et al., 2008).

Group $G$ : The group consists of $G B F$ genes from Arabidopsis and their homologues CPRF1, CPRF3, CPRF4a andCPRF5 are found in parsley. Their function has not been explored properly yet.

Group $\boldsymbol{E}$ : The group E shares a highly similar zipper motif with the members of group I but there is no lysine at position -10 , that's why it has been allotted a different group.

Group H: This group has only two members, AtbZIP56/HY5 and AtbZIP64. HY5 plays role in promoting photomorphogenesis (Table 2) i.e., proper hypocotyl and cotyledon development and expression of light sensitive genes (Jakoby et al., 2002). HY5 responds to a broad range of wavelengths as well as hormones to express downstream genes (Chang et al., 2008). In Arabidopsis light-harvestingchlorophyll A/B (Lhcb) proteins are produced by the genes regulated by circadian clock. Circadian clock associated 1 (CCA-1) is the protein that binds to the CCA-1 binding site (CBS). There is an important interaction between transcription factors binding to the CBS and G-box binding Hy5 proteins in order to conduct a properly rhythmic circadian cycle. G-box core element is found in the Lhcb promoter and physical interface of Hy5 and CCA1 brings about normal circadian expression of Lhcb (Andronis et al., 2008).

Group I: Group I of Arabidopsis bZIP include 13 members that are AtbZIP18, AtbZIP29, AtbZIP30, AtbZIP31, AtbZIP32, AtbZIP33, AtbZIP51, AtbZIP52, AtbZIP59, AtbZIP69, AtbZIP71, AtbZIP73 and AtbZIP74 (Table 1). This group of bZIP transcription factors specifically expresses in stem and controls the activity of gibberellin and hence ultimately controls the height of the plant (Table 2). Disturbance in the function of bZIP group I may result in dwarfism and aberrant stem formation (Jakoby et al., 2002).

Group S: Group S includes four members AtbZIP1, AtbZIP2, AtbZIP44 and AtbZIP53 and are transcriptionally active after stress like drought, cold, wound and/or anaerobiosis (Table 1,2). Group $S$ members of bZIP transcription factors are mainly expressed in basic flower organs. Group $\mathrm{S}$ members are most probably involved in sucrose signaling and control demand and supply of carbohydrates in organisms (Jakoby et al., 2002).

\section{ii. bZIP in cotton:}

Cotton is a major cash crop throughout the world. Intense observations of cotton genome and its expression patterns have revealed that a major part of the genome expresses during the fibre development and ultimately cell wall thickening. There is a tremendous change in the transcripts abundance during genes regulation for fibre development and subsequent metabolism (Al-Ghazi et al., 2009). About 70 
bZIP proteins have been identified in cotton (Gossypium hirsutum spp). Among them GhbZIP was first that was identified by Jiang with his collaborators (2004). GhbZIP has multiple domains and functions as a transcriptional activator in the ovule and fibre cells of cotton, ultimately helping in the fibre elongation of cotton. It has been observed that GhbZIP expresses after three days of anthesis. Sequence analysis of GhbZIP has revealed that it has $24 \%$ sequence similarity with a rice bZIP protein accession number AF268596 and 29\% sequence similarity with Arabidopsis bZIP accession number NM_100091. GhbZIP is a transmembrane protein, according to hydropathy examination, which has its carboxyl end inside the membrane and amino terminal at the outside (Table 1) (Jiang et al., 2004). Four bZIP-like transcription factors have also been detected that are down regulated upon salinity stress (Rodriguez-Uribe et al., 2011).

\section{iii. bZIP in Soybean:}

About 131 bZIP genes have been discovered in Soybean (Glycine max) and termed as GmbZIPs. GmbZIPs are involved in negative signaling of ABA, cold, salt, drought (Liao et al., 2008) and pathogen responsive (Alves et al., 2013). Whole genome shot gun approach was employed by Schmutz and co-workers (2010) to sequence 1.1-gigabase genome of soybean. They generated a chromosome scale draft sequence assembly by using high density genetic maps. They claimed 4630 genes that code various proteins in soybean and this is $70 \%$ bigger than Arabidopsis (Schmutz et al., 2010).

$\boldsymbol{G / H B F - 1 :}$ G/HBF-1 protein is among the very first bZIP proteins characterized in soybean. $\mathrm{G} / \mathrm{HBF}-1$ binds to the ciselements in the H-box and G-box motif, also called pathogen elicitors. Chs 15 is a gene in soybean responsible of producing flavonoids and diterpenoids in response of pathogen attack. Chs 15 promoter contains these G-box and H-box motif. G/HBF-1 gets phosphorylated in the diseased cells unlike $\mathrm{G} / \mathrm{HBF}-1$ proteins and transcript levels that remain constant during the stimulation of chs15 (Alves et al., 2013).

iv. bZIP in tobacco:

$\boldsymbol{R S G}$ : RSG (Repression of Shoot Growth) gene of tobacco shows high resemblance to the Group I of Arabidopsis as it play a role in vascular bundles development. The $R S G$ in tobacco precisely expresses in the phloem and stimulates gibberellin biosynthesis pathway by GA3 gene (Table 1). Experiments on tobacco showed that the production of dominant negative RSG restricted $G A 3$ promoter stimulation, consequently gibberellin production was reduced and dwarf transgenic plants were produced (Jakoby et al., 2002). In tobacco BZI-1 transcription factor forms a protein complex with tobacco ankyrin repeat protein ANK1 which is assumed to be involved in both the auxin-facilitated growth and response to pathogen attack (Schutze et al., 2008).

\section{v. bZIP in tomato:}

$\boldsymbol{V S F - 1 : ~ T h i s ~ i s ~ a ~ m e m b e r ~ o f ~ b Z I P ~ t r a n s c r i p t i o n ~ f a c t o r s ~ w h i c h ~}$ is expressed in vascular tissues and it also stimulates a gene that encodes a structural protein found in the cell wall. VSF1 is the same transcription factor as RF2a in rice and group I in Arabidopsis but there is convergence of its function that provides evidence that some group I bZIPs perform the function of vascular development but there is no proof of such performance by VSF-1 (Jakoby et al., 2002).

\section{vi. bZIPs in rice:}

In rice 89 OsbZIP genes have been identified (Nijhawan et al., 2008). To have a deep discernment into the gene configuration of these 89 OsbZIP genes, their exon/intron arrangement was dissected. Out of 89, 17 (19.1\%) OsbZIP genes have no introns. In the remaining 72 OsbZIP genes in which introns have been detected in ORF have 1-12 numbers of introns. The arrangement of intron locations within the basic and pivot regions of the bZIP domain and intron phases regarding codons are shown in the Figure 2 (Nijhawan et al., 2008). Detailed exploration of OsbZIP has proved that bZIP proteins perform miscellaneous physiological and developmental functions during panicle development, flower development and seed also controls various abiotic pressures and light signaling in rice (Table 2). Detailed knowledge of all functions of OsbZIP has not been unwrapped yet (Nijhawan et al., 2008).

TRAB1: TRAB1 (transcription factor responsible for ABA regulation1) (Schutze et al., 2008) is the group resembles the group $\mathrm{A}$ of Arabidopsis in which $\mathrm{ABA}$ induces the expression of ABI5 protein and its phosphorylation. TRAB1 ABI5 performs the function of recruiting the OsVP1. OsVP1 is an $\mathrm{ABI} 3$ transcriptional factor in rice to the LEA promoter. TRAB1 also plays the role of ABA signal transduction like group-A in Arabidopsis (Jakoby et al., 2002). ABA stress promptly phosphorylates TRAB1 at the point Ser102 into aspartic acid (Umezawa et al., 2006). TRAB1 is phosphorylated due to hyper-osmolarity too; demonstrating that all of the modifications recruited ABA signal pathway during stress conditions (Kagaya et al., 2002). TRAB1 efficiently enhances the transcriptional activity in the rice protoplast transient assay even in the unavailability of inducer ABA (Umezawa et al., 2006). The rice SnRK2 protein kinase family members cause this alteration. In an automated response to ABA, SnRK2 kinase phosphorylates TRAB1 and brings ABRE-controlled reporter gene expression (Schutze et $a l ., 2008)$. OsbZIP have been divided into a-g groups and Intron distribution arrangements are depicted. In the figure above, basic and axis regions have been mentioned. The dissecting arrows figure out introns. The number of OsbZIP proteins and introns are also given. Splicing stages of bZIP domains are also mentioned as $\mathrm{P} 0$ and $\mathrm{P} 2$ 
Table 1. Taxonomy of bZIP gene family of plants and their functions.

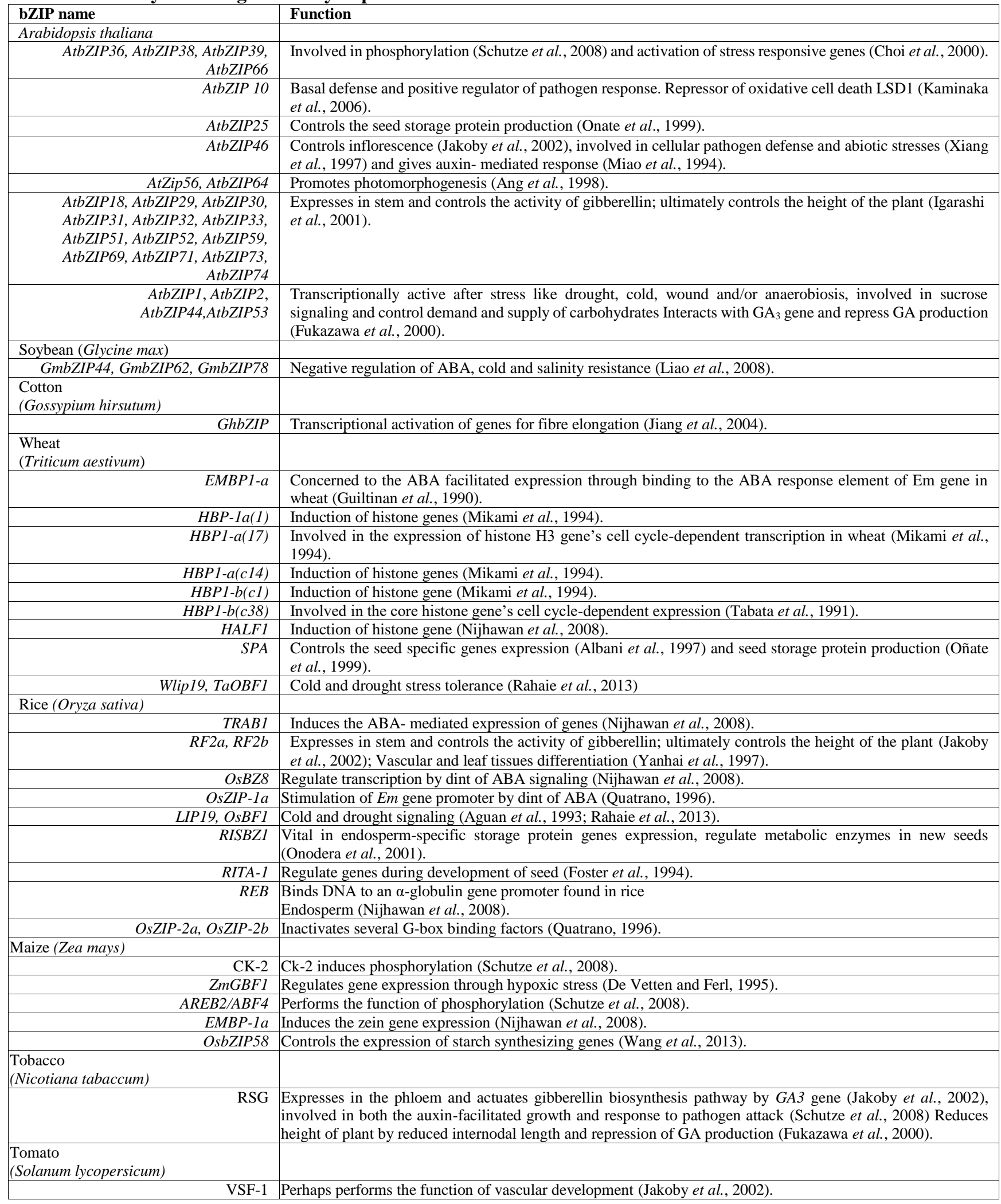


Table 2. Morphological traits associated with different functions regulated by different bZIPs.

\begin{tabular}{|c|c|c|}
\hline Gene name & Functions & Morphological traits \\
\hline$A R E B 1 / A B F 2, A R E B 2 / A B F 4, A B F 3$ & $\begin{array}{l}\text { Involved in phosphorylation } \\
\text { (Schutze et al., 2008) and } \\
\text { activation of stress responsive } \\
\text { genes (Choi } \text { et al., 2000). }\end{array}$ & $\begin{array}{l}\text { Show rigorous growth (Bartels and } \\
\text { Sunkar, 2005) and high survivability in } \\
\text { stress conditions (Fujita et al., 2005). }\end{array}$ \\
\hline AtbZIP1, AtbZIP2, AtbZIP44, AtbZIP53 & $\begin{array}{l}\text { Transcriptionally active after } \\
\text { stress like drought, cold, wound } \\
\text { and/or anaerobiosis, involved in } \\
\text { sucrose signaling and control } \\
\text { demand and supply of } \\
\text { carbohydrates (Fukazawa et al., } \\
\text { 2000). }\end{array}$ & $\begin{array}{l}\text { Stomatal closure at daytime (Yordanov } \\
\text { et al., 2003), adopt thick leaves with } \\
\text { hairs and waxiness. } \\
\text { Many drought-tolerant species have } \\
\text { thick, waxy, or hairy leaves that } \\
\text { minimize water loss (Shinozaki et al., } \\
\text { 2007). }\end{array}$ \\
\hline$L I P 19$, OsBF1 & $\begin{array}{l}\text { Cold signaling(Aguan et al., } \\
\text { 1993). }\end{array}$ & $\begin{array}{l}\text { More spikelets, awn less plants, } \\
\text { reduced height and biomass, longer } \\
\text { panicle but lower seed set. (James, } \\
\text { 2012) }\end{array}$ \\
\hline $\begin{array}{l}\text { AtbZIP18, AtbZIP29, AtbZIP30, AtbZIP31, } \\
\text { AtbZIP32, AtbZIP33, AtbZIP51, AtbZIP52, } \\
\text { AtbZIP59, AtbZIP69, AtbZIP71, AtbZIP73, } \\
\text { AtbZIP74 }\end{array}$ & $\begin{array}{l}\text { Expresses in stem and controls the } \\
\text { activity of gibberellin; ultimately } \\
\text { controls the height of the plant } \\
\text { (Igarashi } \text { et al., 2001). }\end{array}$ & $\begin{array}{l}\text { Over expression increase the } \\
\text { vegetative growth and plant height } \\
\text { whereas some mutants show reduced } \\
\text { response to gibberellins (Radi et al., } \\
\text { 2006). }\end{array}$ \\
\hline AtbZIP46 & $\begin{array}{l}\text { Controls inflorescence (Jakoby et } \\
\text { al., 2002). }\end{array}$ & $\begin{array}{ll}\begin{array}{l}\text { Results in pentamerous flowers } \\
\text { (Chuang } \text { et al., 1999). }\end{array} & \\
\end{array}$ \\
\hline AtZip56, AtbZIP64 & $\begin{array}{l}\text { Promotes photomorphogenesis } \\
\text { (Ang } \text { et al., 1998). }\end{array}$ & $\begin{array}{l}\text { Below par development of cotyledons } \\
\text { and enlarged hypocotyl (Jakoby } \text { et al., } \\
\text { 2002). Reduced lateral roots } \\
\text { development (Ang } \text { et al., 1998). }\end{array}$ \\
\hline GhbZIP & $\begin{array}{l}\text { Transcriptional activation of genes } \\
\text { for fibre elongation (Jiang et al., } \\
2004 \text { ). }\end{array}$ & Fibre elongation (Jiang et al., 2004). \\
\hline$R F 2 a, R F 2 b$ & $\begin{array}{l}\text { Expresses in stem and controls the } \\
\text { activity of gibberellin; ultimately } \\
\text { controls the height of the plant } \\
\text { (Jakoby et al., 2002) Vascular and } \\
\text { leaf tissues differentiation(Yanhai } \\
\text { et al., 1997). }\end{array}$ & $\begin{array}{l}\text { Produce dwarf plants and anomalous } \\
\text { vascularization (Jakoby et al., 2002). }\end{array}$ \\
\hline OsbZIP58 & $\begin{array}{l}\text { Controls the expression of starch } \\
\text { synthesizing genes (Wang et al., } \\
\text { 2013). }\end{array}$ & $\begin{array}{l}\text { Excellent quality grain and mutants } \\
\text { show abnormal seed formation with } \\
\text { low starch (Wang et al., 2013) }\end{array}$ \\
\hline
\end{tabular}




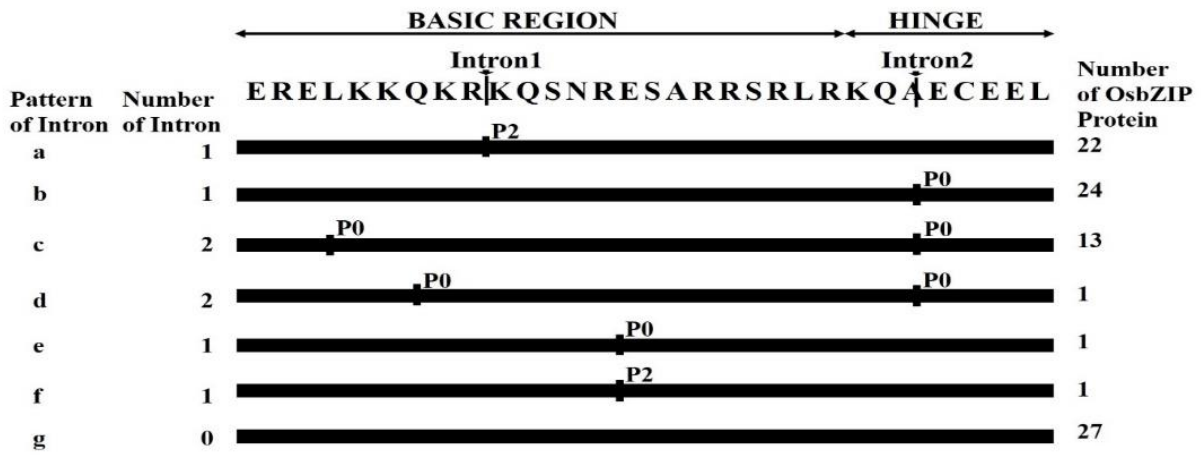

Figure 2. BZIP domains of OsbZIP showing Intron distribution.

$\boldsymbol{R F} 2$ : RF2A has been identified in rice which is similar to the bZIP I group in Arabidopsis. Suppression lines of $R F 2 a$ antisense gene have been developed in rice which exhibit short stature phenotype. This phenomenon might be due to suppression in biosynthesis pathway of gibberellin (Figure 3). Moreover, these plants show peculiar development of vascular tissue (Jakoby et al., 2002).

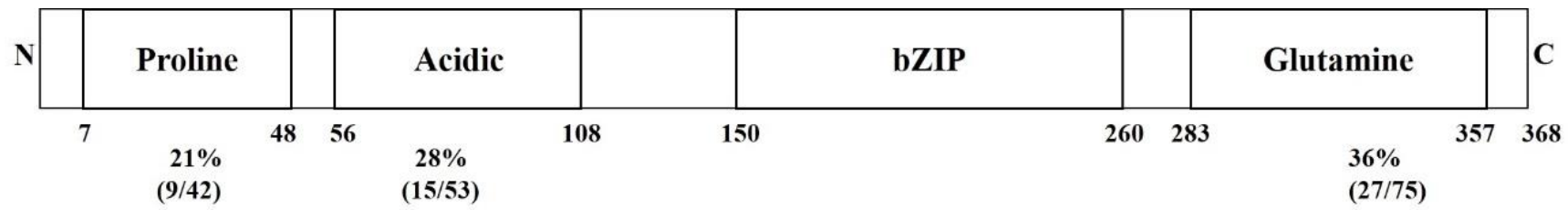

Figure 3. Domain structure of RF2a with percentages of proline, acidic amino acids and glutamine residues in each presumed domain.

There is an important viral disease of rice caused by rice tungro bacilliform virus (RTBV) which replicates in infected phloem cells of rice plants. In rice plants, promoter of RTBV induces a reporter gene expression. RF2a encodes for a basic leucine zipper protein and binds to Box II cis element. Box II cis element is a compulsory requirement for the promoter expression of RTBV. RF2a is mostly found in phloem cells, shoots and very small amount in roots and rouses Box II hooked transcription in homologous in vitro system of transcription. In transgenic antisense plants in which RF2a amassing was bottled-up had normal roots but underdeveloped and curled leaves with small, disordered vascular tissues, bloated sclerenchyma and outsized air spaces. Hence it was concluded that a host transcription factor has been exploited by the RTBV promoter that is vital for differentiation of leaves and vascular tissues development (Yanhai et al., 1997).

OsbZIP-1a: OsbZIP-1a is a homolog of EMBP-1 and binds to the G-box in the DNA sequence. OsbZIP-1a is found in the rice protoplast and induces Em promoter through $\mathrm{ABA}$ induction. There is a P-ring at its amino terminal which is referred as ATP/GTP binding domain (Quatrano, 1996).

OsbZIP58: OsbZIP monitors the expression of starch synthesis genes in rice i.e. ISA2, OsSSIIa, Wx, OsBEIIb, OsAGPL3, SBE1; by directly attaching to their promoters and help in production of high quality starch in seeds. In the absence of OsbZIP58 the rice grain becomes deformed with lower quantity of amylose and starch and aggregation of a different type of starch in the belly portion (Table 2). OsbZIP58 is a good indicator of high quality rice plant (Wang et al., 2013).

$V \boldsymbol{V F}$-1: VSF-1 is a bZIP transcription factor that binds to the promoters of grp 1.8 genes. VSF-1 rouses $\operatorname{grp} 1.8$ transcription in protoplast (Yanhai et al., 1997).

vii. bZIP in wheat:

HBP1a: Histone binding protein 1a (HBP1a) is bZIP which particularly bind to the ACGT core sequence of promoter. HBP-1 has a characteristic bZIP domain at C-terminal and a proline rich domain at $\mathrm{N}$-terminal and bZIP domain binds to the CCACGT motif. HBP1 is further divided into sub groups e.g., HBP1a-1, HBP1a-17 and HBP1a-c14. All members of HBP1 binds to the same CCACGT motif but their binding tendencies are diverse. HBP1a is specific to bind to the $\mathrm{H} 3$ promoter that is a hexamer motif. HBP1a-17 aggregates at meristematic tissues, in which the $S$-phase is plentiful. Thus it is assumed that the cell cycle dependent transcription of $\mathrm{H} 3$ gene is controlled by HBP1a-17. HBP1a-17 makes a homodimers with the hexamer motif and it is also involved in the activation of the CTF domain in humans and GBF1 
domain in Arabidopsis thaliana.

Keeping in view a great variety of functions of the members, HBP1a has been categorized into various sub groups i.e., common plant regulatory factors as CPRF-1, CPRF-3, G-Box binding factors as GBFs and TGACGT core binding as TGAlb. Hence it can be assumed that a separate superfamily of HBP-1a could be found in various plant species (Mikami et al., 1994).

HBP1b: HBP1b is the second subclass of histone binding protein. This is an isoform of HBP1a but binds to a different hexamer motif i.e. ACGTCA, also termed as Hex-b motif. Both HBP1a and HBP1b have the same bZIP domain but in contrast to HBP1a, there are glutamine residues at C-terminal in HBP1b. The binding specificities of HBP1a and HBP1b are defined by the flanking core sequence of the hexamer as the ACGT core is common for both. In some plants and animals, some other transcription factors also act as trans-activation domain. HBP1b-c38 also gathers at meristematic tissues of plant in which S-phase is abundant, hence controlling the cell cycle dependent transcription of $\mathrm{H} 3$ gene in wheat.

HBP1-b has also some recognized proteins like vitellogenin gene binding protein (VBP1), TGA1 and TGAla. Hence HBP1b can also contribute towards the new expected superfamily of gene termed as Histone binding proteins (Mikami et al., 1994).

Wlip19: Wheat lip19 is a cold, drought and ABA responsive bZIP-type transcription factor with an exclusively higher expression in cold tolerant cultivars of wheat than coldsensitive genotypes. Wlip19 mends the expression of four Cor/Lea genes of wheat named Wrab17, Wrab18, Wrab19 and Wdhn13 of wheat callus to impose stress tolerance. Its heterolog in tobacco shows major tolerance to abiotic stresses specifically cold. Even Wlip19 shows its expression upon exogenous application of ABA (Kobayashi et al., 2008). Further investigation has showed that Wlip19 and another bZIP type Transcription factor TaOBF1 has same proteinprotein interface in all cereals (Rahaie et al., 2013).

TaABF1: Autonomously replicating sequence i.e. ARSbinding factor (ABF1) in wheat is a seed specific gene (Johnson et al., 2002), involved in ABA stimulated regulation of HVAl gene in wheat in a non-additive manner and expresses in the seeds during imbibition (Keyser, 2010). TaABF1 is not expressed vegetative tissues of plant neither upon ABA application nor in response to abiotic stress (Johnson et al., 2002; Rahaie et al., 2011). Detailed exploration of mechanism of action of TaABF1 revealed that during maturation and dormancy development of wheat grain mRNA of TaABF1 along with an ABA-induced protein kinase (PKABA1) mount up in seeds. Transcripts of TaABF1 tremendously add up in seed during imbibition (Rahaie et al., 2011). TaABF1 is not responsive to abiotic stresses but its homologous bZIP1 shows response to salinity by down regulation (Rahaie et al., 2013).
SPA: Storage protein activator (SPA) is a unique transcription factor of wheat, particular to seed storage proteins. SPA is analogous to the $\mathrm{O}_{2}$ transcription factor in maize. Endosperm motif (EM) is an essential requirement for the transcriptional activity of SPA. SPA activates the reporter gene by binding to the long endosperm box (LEB) and $\mathrm{GCN}_{4}$ like motif (GLM) (Albani et al., 1997).

\section{viii. bZIP in maize:}

CK2: The group A-bZIP undergoes phosphorylation induced by ABA. CK2 protein kinase in maize phosphorylates two maize bZIP factors. CK2-induced phosphorylation enhances the DNA binding process of EmBP-2 and lowers that of ZmBZ-1 (Schutze et al., 2008).

AREB2/ABF4: There is a protein kinase dependent on calcium, AtCPK32 that phosphorylates AREB2 at Ser 110 position in vitro. This residue is necessary for ABF4 transcriptional activity too which depicts the straightforward contribution of AtCPK32 in ABA-responsive gene manifestation (Schutze et al., 2008).

SPA: SPA is involved in $22-\mathrm{kD}$ zein gene transcription. SPA and $\mathrm{O} 2$ have similar sequence to some extent but their transcriptional activity potential is same (Albani et al., 1997). Conclusions: Plant bZIPs show a grand diversity in nature like DNA binding and transcriptional activities, ability to form homodimers and heterodimers and interaction with proteins other than leucine zipper proteins. Its diversity of activities makes it adaptive to a variety of stresses and responds accordingly in both forward and reverse manner. Most of the higher plants bZIP proteins depend upon the hexamer motif in DNA region for binding to the particular transcription factor. All bZIP proteins show binding specificity to the hexamer motif in DNA which gives a clear cut view of the importance of the bZIP transcription factors for higher plants. These traits make the bZIP transcription factors more complex and finely tuned and still there is a huge potential yet to be disclosed. Here we have given a classification of bZIP transcription factors according to their binding specificities as well as their functions but still there is a great range of unknown bZIP transcription factors which need to be allotted their special place in any of these groups or new.

\section{REFERENCES}

Aguan, K., K. Sugawara, N. Suzuki and T. Kusano. 1993. Low-temperature-dependent expression of a rice gene encoding a protein with a leucine-zipper motif. Mol. Gen. Genet. 240:1-8.

Albani, D., M. Hammond-Kosack, C. Smith, S. Conlan, V. Colot, M. Holdsworth and M.W. Bevan. 1997. The wheat transcriptional activator SPA: a seed-specific bZIP protein that recognizes the GCN4-like motif in the bifactorial endosperm box of prolamin genes. Plant Cell 9:171-184. 
Al-Ghazi, Y., S. Bourot, T. Arioli, E.S. Dennis and D.J. Llewellyn. 2009. Transcript profiling during fiber development identifies pathways in secondary metabolism and cell wall structure that may contribute to cotton fiber quality. Plant Cell Physiol. 50:1364-1381.

Allen, R.D. 2012. Evaluation of drought tolerance strategies in cotton. Ntl. Agri. Biotech. Council; pp.45-63.

Alves, M.S., S.P. Dadalto, A.B. Gonçalves, G.B. De Souza, V.A. Barros and L.G. Fietto. 2013. Plant bZIP transcription factors responsive to pathogens: a review. Intl. J. Mol. Sci. 14:7815-7828.

Andronis, C., S. Barak, S.M. Knowles, S. Sugano and E.M. Tobin. 2008. The clock protein CCA1 and the bZIP transcription factor HY5 physically interact to regulate gene expression in Arabidopsis. Mol. Plant 1:58-67.

Ang, L.H., S. Chattopadhyay, N. Wei, T. Oyama, K. Okada, A. Batschauer and X.W. Deng. 1998. Molecular interaction between COP1 and HY5 defines a regulatory switch for light control of Arabidopsis development. Mol. Cell 1:213-222.

Bartels, D. and R. Sunkar. 2005. Drought and salt tolerance in plants. Crit. Rev. Plant Sci. 24:23-58.

Chang, C.S.J., Y.H. Li, L.T. Chen, W.C. Chen, W.P. Hsieh, J. Shin, W.N. Jane, S.J. Chou, G. Choi and J.M. Hu. 2008. LZF1, a HY5-regulated transcriptional factor, functions in Arabidopsis de-etiolation. Plant J. 54:205-219.

Cheong, Y.H., B.C. Moon, J.K. Kim, C.Y. Kim, M.C. Kim, I.H. Kim, C.Y. Park, J.C. Kim, B.O. Park and S.C. Koo. 2003. BWMK1, a rice mitogen-activated protein kinase, locates in the nucleus and mediates pathogenesis-related gene expression by activation of a transcription factor. Plant Physiol. 132:1961-1972.

Chinnusamy, V., K. Schumaker and J.K. Zhu. 2004. Molecular genetic perspectives on cross-talk and specificity in abiotic stress signaling in plants. J. Exp. Bot. 55:225-236.

Choi, H.I., J.H. Hong, J.O. Ha, J.Y. Kang and S.Y. Kim. 2000. ABFs, a family of ABA-responsive element binding factors. J. Biol. Chem. 275:1723-1730.

Chuang, C.F., M.P. Running, R.W. Williams and E.M. Meyerowitz. 1999. The PERIANTHIA gene encodes a bZIP protein involved in the determination of floral organ number in Arabidopsis thaliana. Genes Dev. 13:334-344.

De Vetten, N.C. and R.J. Ferl. 1995. Characterization of a maize G-box binding factor that is induced by hypoxia. Plant J. 7: 589-601.

Fujita, Y., M. Fujita, R. Satoh, K. Maruyama, M.M. Parvez, M. Seki, K. Hiratsu, M. Ohme-Takagi, K. Shinozaki and K. Yamaguchi-Shinozaki. 2005. AREB1 is a transcription activator of novel ABRE-dependent ABA signaling that enhances drought stress tolerance in Arabidopsis. Plant Cell 17:3470-3488.
Fukazawa, J., T. Sakai, S. Ishida, I. Yamaguchi, Y. Kamiya and Y. Takahashi. 2000. Repression of shoot growth, a bZIP transcriptional activator, regulates cell elongation by controlling the level of gibberellins. Plant Cell 12:901915.

Guiltinan, M.J., W.R. Marcotte and R.S. Quatrano. 1990. A plant leucine zipper protein that recognizes an abscisic acid response element. Sciences 250:267-271.

Holdsworth, M.J., J. Muñoz-Blanco, M. Hammond-Kosack, V. Colot, W. Schuch and M.W. Bevan. 1995. The maize transcription factor Opaque-2 activates a wheat glutenin promoter in plant and yeast cells. Plant Mol. Biol. 29:711720.

Igarashi, D., S. Ishida, J. Fukazawa and Y. Takahashi. 2001. 14-3-3 proteins regulate intracellular localization of the bZIP transcriptional activator RSG. Plant Cell 13:24832497.

Jakoby, M., B. Weisshaar, W. Dröge-Laser, J. VicenteCarbajosa, J. Tiedemann, T. Kroj and F. Parcy. 2002. bZIP transcription factors in arabidopsis. Trends Plant Sci. 7:106-111.

James, M. 2012. Transcription factors important in the regulation of salinity tolerance.

Jiang, J., W. Guo and T. Zhang. 2004. Cloning and expression analysis of a bZIP cDNA in Gossypium hirsutum L. Yi chuan xue bao = Acta gen. Sin. 31:616-621.

Johnson, R.R., R.L. Wagner, S.D. Verhey and M.K. WalkerSimmons. 2002. The abscisic acid-responsive kinase PKABA1 interacts with a seed-specific abscisic acid response element-binding factor, TaABF, and phosphorylates TaABF peptide sequences. Plant Physiol. 130:837-846.

Kagaya, Y., T. Hobo, M. Murata, A. Ban and T. Hattori. 2002. Abscisic acid-induced transcription is mediated by phosphorylation of an abscisic acid response element binding factor, TRAB1. Plant Cell 14:3177-3189.

Kaminaka, H., C. Nake, P. Epple, J. Dittgen, K. Schutze, C. Chaban, B.F. Holt, T. Merkle, E. Schafer and K. Harter. 2006. bZIP10-LSD1 antagonism modulates basal defense and cell death in arabidopsis following infection. EMBO J. 25:4400-4411.

Kang, J.Y., H.I. Choi, M.Y. Im and S.Y. Kim. 2002. Arabidopsis basic leucine zipper proteins that mediate stress-responsive abscisic acid signaling. Plant Cell 14:343-357.

Keyser, B.R. 2010. The wheat bZIP factor, TaABF1, mediates ABA-induced gene expression in bombarded barley aleurone layers. Honors Theses, Colby College Department of Biology; Paper 593. http:// digitalcommons.colby.edu/honorstheses/593

Kobayashi, F., E. Maeta, A. Terashima, K. Kawaura, Y. Ogihara and S. Takumi. 2008. Development of abiotic 
stress tolerance via bZIP-type transcription factor LIP19 in common wheat. J. Exp. Bot. 59:891-905.

Liao, Y., H.F. Zou, W. Wei, Y.J. Hao, A.-G. Tian, J. Huang, Y.F. Liu, J.S. Zhang and S.Y. Chen. 2008. Soybean GmbZIP44, GmbZIP62 and GmbZIP78 genes function as negative regulator of $\mathrm{ABA}$ signaling and confer salt and freezing tolerance in transgenic Arabidopsis. Planta 228:225-240.

Miao, Z.H., X. Liu and E. Lam. 1994. TGA3 is a distinct member of the TGA family of bZIP transcription factors in Arabidopsis thaliana. Plant Mol. Biol. 25:1-11.

Mikami, K., A. Sakamoto and M. Iwabuchi. 1994. The HBP1 family of wheat basic/leucine zipper proteins interacts with overlapping cis-acting hexamer motifs of plant histone genes. J. Biol. Chem. 269:9974-9985.

Nakashima, K., Y. Ito and K. Yamaguchi-Shinozaki. 2009. Transcriptional regulatory networks in response to abiotic stresses in Arabidopsis and grasses. Plant Physiol. 149:8895.

Narusaka, Y., K. Nakashima, Z.K. Shinwari, Y. Sakuma, T. Furihata, H. Abe, M. Narusaka, K. Shinozaki and K. Yamaguchi-Shinozaki. 2003. Interaction between two cisacting elements, ABRE and DRE, in ABA-dependent expression of arabidopsis $R d 29 \mathrm{~A}$ gene in response to dehydration and high salinity stresses. Plant J. 34:137148.

Nijhawan, A., M. Jain, A.K. Tyagi and J.P. Khurana. 2008. Genomic survey and gene expression analysis of the basic leucine zipper transcription factor family in rice. Plant Physiol. 146:333-350.

Onate, L., J. Vicente-Carbajosa, P. Lara, I. Diaz and P. Carbonero. 1999. Barley BLZ2, a seed-specific bZIP protein that interacts with BLZ1 in vivo and activates transcription from the GCN4-like motif of B-hordein promoters in barley endosperm. J. Biol. Chem. 274:91759182.

Onodera, Y., A. Suzuki, C.-Y. Wu, H. Washida and F. Takaiwa. 2001. A rice functional transcriptional activator, RISBZ1, responsible for endosperm-specific expression of storage protein genes through GCN4 motif. J. Biol. Chem. 276:14139-14152.

Plant Transcription Factor Database. Centre for Bioinformatics, Peking University, China. Available online at http://planttfdb.cbi.pku.edu.cn/index.php

Quatrano, N.A.R.S. 1996. Characterization of three rice basic/leucine zipper factors, including two inhibitors of EmBP-1 DNA Binding Activity. J. Biol. Chem. 271:31296-31305.

Radi, A., T. Lange, T. Niki, M. Koshioka and M.J.P. Lange. 2006. Ectopic expression of pumpkin gibberellin oxidases alters gibberellin biosynthesis and development of transgenic Arabidopsis plants. Plant Physiol. 140:528536.
Rahaie, M., G.-P. Xue and P.M. Schenk. 2013. The role of transcription factors in wheat under different abiotic stresses. Development 2:59. doi: 10.5772/54795

Rahaie, M., G.P. Xue, M.R. Naghavi, H. Alizadeh and P.M. Schenk. 2010. A MYB gene from wheat (Triticum aestivum L.) is up-regulated during salt and drought stresses and differentially regulated between salt-tolerant and sensitive genotypes. Plant Cell Rep. 29:835-844.

Rahaie, M., M. Gomarian, H. Alizadeh, M. Malboobi and M. Naghavi. 2011. The expression analysis of transcription factors under long term salt stress in tolerant and susceptible wheat genotypes using reverse northern blot technique. Iranian J. Crop Sci. 13:580-595.

Rodriguez-Uribe, L., S.M. Higbie, J.M. Stewart, T. Wilkins, W. Lindemann, C. Sengupta-Gopalan and J. Zhang. 2011. Identification of salt responsive genes using comparative microarray analysis in upland cotton (Gossypium hirsutum L.). Plant Sci. 180:461-469.

Schmutz, J., S.B. Cannon, J. Schlueter, J. Ma, T. Mitros, W. Nelson, D.L. Hyten, Q. Song, J.J. Thelen and J. Cheng. 2010. Genome sequence of the palaeopolyploid soybean. Nature 463:178-183.

Schutze, K., K. Harter and C. Chaban. 2008. Posttranslational regulation of plant bZIP factors. Trends Plant Sci. 13:247-255.

Seldeen, K.L., C.B. McDonald, B.J. Deegan, V. Bhat and A. Farooq. 2010. Dissecting the role of leucine zippers in the binding of bZIP domains of Jun transcription factor to DNA. Biochem. Biophys. Res. Commun. 394:1030-1035.

Selvaraj, D., A. Loganathan and R. Sathishkumar. 2010. Molecular characterization and phylogenetic analysis of bZIP protein in plants. J. Proteomics Bioinform. 3:230233.

Shen, Q. and T. Ho. 1995. Functional dissection of an abscisic acid (ABA)-inducible gene reveals two independent ABA-responsive complexes each containing a G-box and a novel cis-acting element. Plant Cell 7:295-307.

Shinozaki, K. and K. Yamaguchi-Shinozaki. 2007. Gene networks involved in drought stress response and tolerance. J. Exp. Bot. 58:221-227.

Shinozaki, K., K. Yamaguchi-Shinozaki and M. Seki. 2003. Regulatory network of gene expression in the drought and cold stress responses. Curr. Opin. Plant Biol. 6:410-417.

Singh, K.B., R.C. Foley and L. Onate-Sanchez. 2002. Transcription factors in plant defense and stress responses. Curr. Opin. Plant Biol. 5:430-436.

Tabata, T., T. Nakayama, K. Mikami and M. Iwabuchi. 1991. $H B P-1 a$ and $H B P-1 b$ : leucine zipper-type transcription factors of wheat. EMBO J. 10:1459-1468.

Umezawa, T., M. Fujita, Y. Fujita, K. Yamaguchi-Shinozaki and K. Shinozaki. 2006. Engineering drought tolerance in plants: discovering and tailoring genes to unlock the future. Curr. Opin. Biotech. 17:113-122. 
Vincentz, M., P.S. Schlogl, L.G.G. Correa, F. Kuhne and A. Leite. 2001. Phylogenetic relationships between arabidopsis and sugarcane bZIP transcriptional regulatory factors. Genet. Mol. Biol. 24:55-60.

Wang, J.C., H. Xu, Y. Zhu, Q.Q. Liu and X.L. Cai. 2013. OsbZIP58, a basic leucine zipper transcription factor, regulates starch biosynthesis in rice endosperm. J. Exp. Bot. 64:3453-3466.

Wei, L.Q., W.Y. Xu, Z.Y. Deng, Z. Su, Y. Xue and T. Wang. 2010. Genome-scale analysis and comparison of gene expression profiles in developing and germinated pollen in Oryza sativa. BMC Genomics 11:338. doi: 10.1186/1471-2164-11-338.

Xiang, C., Z. Miao and E. Lam. 1997. DNA-binding properties, genomic organization and expression pattern of TGA6, a new member of the TGA family of bZIP transcription factors in Arabidopsis thaliana. Plant Mol. Biol. 34:403-415.
Yin, Y., Q. Zhu, S. Dai, C. Lamb and R.N. Beachy. 1997. RF2a, a bZIP transcriptional activator of the phloemspecific rice Tungro bacilliform virus promoter, functions in vascular development. EMBO J. 16:5247-5259.

Yordanov, I., V. Velikova and T. Tsonev. 2000. Plant responses to drought, acclimation, and stress tolerance. Photosynthetica 38:171-186.

Zhang, L., G. Zhao, J. Jia, X. Liu and X. Kong. 2012. Molecular characterization of 60 isolated wheat MYB genes and analysis of their expression during abiotic stress. J. Exp. Bot. 63:203-214.

Zhang, Y., G. Zhang, N. Xia, X.J. Wang, L.L. Huang and Z.S. Kang. 2008. Cloning and characterization of a bZIP transcription factor gene in wheat and its expression in response to stripe rust pathogen infection and abiotic stresses. Physiol. Mol. Plant Pathol. 73:88-94. 